\title{
Descontruyendo las masculinidades hegemónicas - un medio para la prevención de la Violencia Basada en el género en las comunidades de El Viejo - Chinandega.
}

\author{
Deconstructing hegemonic masculinities - a means for the prevention of gender-based violence in the \\ communities of El Viejo - Chinandega
}

Sergio José Hernández Briceño

Recibido: 25-08-2018

Antropólogo - Consultor independiente

ID Orcid: https://orcid.org/0000-0002-2276-383X

hsbjose@gmail.com

\section{Resumen}

El presente artículo es elaborado a partir del involucramiento del autor durante los últimos 3 años (2016-2018) en acciones e intervenciones de carácter comunitario donde se promueven procesos para el cambio social en pro de la equidad y la igualdad de género, por ello el título: "Descontruyendo las masculinidades hegemónicas - un medio para la prevención de la Violencia Basada en el género”. Apunta rescatar algunos elementos extraídos desde la experiencia del trabajo con hombres de diversas edades, quienes han tenido que asumir desafíos para lograr cambios reales que aporten al desarrollo de sí mismos, sus compañeras, familias y espacios comunitarios.

El propósito de este artículo investigativo es dar a conocer la importancia que tiene el trabajo con hombres para fomentar la sensibilización, concientización de género, esto para gestar cambios consecuentes en las relaciones de los hombres con sus familias, promoviendo la no violencia y la afectividad sana; para ello se deben llevar a cabo acciones que cuestionen mandatos asignados por la misma sociedad en donde las relaciones de poder favorecen a los hombres, relegando consecuentemente a las mujeres hacia situaciones de violencia y sumisión.

El tema ha sido abordado desde el enfoque holístico- antropológico, con métodos propias como la etnografía y técnicas particulares de la ciencia como la observación directa y participante, aplicación de entrevistas abiertas y a profundidad.

Palabras claves: Sistema patriarcal, Masculinidades, familias, sociedad, Violencia.

\section{Abstract}

This article is based on the involvement of the author during the last 3 years (2016-2018) in community actions and interventions where processes for social change are promoted in favor of equity and gender equality, therefore the title: «Deconstructing hegemonic masculinities - a means for the prevention of gender-based violence». It aims to rescue some elements extracted from the experience of working with men of different ages, who have had to assume challenges to achieve real changes that contribute to the development of themselves, their companions, families and community spaces.

The purpose of this research article is to make known the importance of working with men to promote awareness, gender awareness, this to gestate consequent changes in the relationships of men with their families, promoting nonviolence and healthy affectivity ; To this end, actions must be carried out that question mandates assigned by the same society in which power relations favor men, consequently relegating women to situations of violence and submission.

The subject has been approached from the holistic-anthropological approach, with its own methods such as ethnography and particular techniques of science such as direct and participant observation, application of open and in-depth interviews.

Keywords: Patriarchal system, Masculinities, families, society, Violence. 


\section{Introducción}

El trabajo con hombres representa una forma más que novedosa, necesaria para aportar a la equidad e igualdad entre mujeres y hombres en los distintos estratos sociales; a través del trabajo con hombres es posible aminorar patrones socioculturales que refuerzan comportamientos trasgresores y violentos desde el ejercicio de roles masculinos en los escenarios familiares y sociales. Por todo esto fomentar procesos reales de cambios en los actores masculinos es una ventana para divisar alternativas de un desarrollo integral en la sociedad.

Llevar a cabo planes y políticas personales concretas que contradigan las acciones de violencia en la familia y sociedad representa un resultado a largo plazo desde los procesos trabajados con hombres, sin embargo al partir desde una línea de sensibilización se incluye precisamente asumir actos que disminuyan estratégicamente las desigualdades y la propia violencia basada en género.

El enfoque de género para trabajar la prevención de la Violencia en Latinoamérica dio sus primeros pasos directamente con las mujeres, quienes atendiendo a su demanda por empoderarse y emanciparse del sistema patriarcal, fueron generando cambios, adoptando nuevas actitudes y demandando iguales escenarios de derecho desde su condición de persona.

Sin embargo los efectos del sistema desventajoso para las mujeres iban más allá de su condición individual, vinculando directamente a su entorno y los actores de este, es ahí donde la labor con hombres se convierte en una nueva estrategia de trabajo para alcanzar niveles deseables de igualdad y equidad de género en la sociedad.

Tomando en cuenta las variables de equidad e igualdad, resulta oportuno valorar que el PNUD define la igualdad de género como "una meta en sí misma, instalada en la base del desarrollo humano y los derechos humanos, requiere de la institucionalización del género y el empoderamiento de las mujeres" (PNUD, 2005),

Es precisamente en la línea del empoderamiento de las mujeres que las figuras masculinas influyen directamente en el desarrollo del mismo, es básicamente irreal mencionar la palabra desarrollo si las prácticas cotidianas se continúa relegando a la mujer.
De igual modo el Programa de las Naciones Unidas para el Desarrollo (PNUD), retoma la variable de equidad de género y la define acorde a sus consideraciones como.

\begin{abstract}
Una exigencia normativa y un requisito para el desarrollo sustentable en el marco del enfoque del Desarrollo Humano. Las desigualdades de género requieren de una potenciación social de las mujeres: es decir, su incorporación equitativa a las instancias de poder económico, social y político (PNUD, 2005)
\end{abstract}

Es claro que la complejidad existente al hablar de la equidad de género en consideración a cada una de sus dimensiones, representa una meta amplia cuyo requerimiento para su debido alcance es la inclusión clave de diversos actores sociales en los escenarios sociopolíticos y económicos.

En el trabajo con hombres se promueven las masculinidades en positivo, mismas que se contraponen a las clásicas masculinidades opresoras y transgresoras de derechos. Es por ello que en el presente estudio se pretende abordar a raíz de la experiencia de trabajo en comunidades rurales de El Viejo - Chinandega, algunos mitos, estigmas y estereotipos que tienen los hombres de la zona y los cuales condicionan el tipo de relaciones de estos individuos con actoras (es) de su entorno, se analiza el trabajo con hombres como una oportunidad de combatir los efectos del sistema patriarcal y tener la oportunidad de lograr con ello alcances reales y equitativos para el desarrollo integral.

\section{Metodologia}

El estudio Descontruyendo las masculinidades hegemónicas - un medio para la prevención de la Violencia Basada en el género en las comunidades de El Viejo - Chinandega. Emerge a partir del desarrollo de diversas experiencias en el área comunitaria en los últimos 3 años (2016 - 2018), tiempo en la que se ha podido trabajar con hombres de diversas edades pertenecientes a las zonas rurales del municipio de El Viejo y quienes evidencian patrones socioculturales inculcados desde un sistema patriarcal, donde son reafirmadas consignas que favorecen al segmento masculino y aminoran las capacidades y destrezas de los grupos de féminas. 
En la recolección y análisis de datos cualitativos y cuantitativos fue posible extraer insumos que detallan una amalgama de acciones y pensamientos propios de los hombres, mismos productos de los efectos que tienen los patrones de comportamiento machitas siendo elementos obstaculizadores en la gestión e implementación de estrategias que promuevan el desarrollo individual y familiar dentro de un entorno saludable y seguro.

Para el desarrollo del presente artículo se hizo uso del método etnográfico y de las técnicas propias de la investigación antropológica. El método etnográfico "consiste en descripciones detalladas de situaciones, eventos, personas, interacciones y comportamientos que son observables" (González, 2003). Siendo de gran utilidad para un proceso descriptivo y consecuente análisis de la realidad actual de las familias y comunidades en estudio, quienes han moldeado patrones conductuales que reflejan la vivencia e incluso la normalización de una cultura no violenta en los espacios de desarrollo inmediato.

De igual manera se aplicó la técnica de observación directa, definida por Marshall y Rossman (1989) como aquella que "consiste en la descripción sistemática de evento, comportamiento y artefactos en el escenario social elegido para ser estudiado" (Marshall, 1989, pág. 7). Al emplear esta técnica se contempló el comportamiento de los hombres en las comunidades en sus escenarios familiares y comunales, se divisó el desarrollo de sus actos guiados por un imaginario social que les refuerza sus roles y posiciones afines a la superioridad y consecuente sumisión de las mujeres, así como el análisis de expresiones coloquiales, fraseos y reproducción de estereotipos productos del mismo sistema patriarcal.

Se empleó la técnica de entrevistas abiertas y a profundidad dirigida a actores sociales, comunitarios. Los criterios de selección consistían en que estas actoras y actores pudieran haber vivenciado alguna situación de violencia o bien conocían a otra persona que si había pasado por alguna circunstancias de este tipo en la comunidad.

Al mismo tiempo se aplicó un test de entrada sobre mitos en las relaciones entre mujeres y hombres, en el cual se extrajo una muestra de los pensamientos que tienen muchos hombres en el marco de las relaciones de género, este test se aplicó a 63 hombres de 10 comunidades rurales de El Viejo, quienes sostenían relaciones de pareja siendo referentes afectivos de sus hogares en las comunidades.

Al mismo tiempo se tomaron a consideración espacios de conversación de carácter informal que dieron aportes a que los hombres se desarrollaran un ambiente de mayor confianza, esto contribuyó a una consecuente eficiencia investigativa con los mismos hombres quienes brindaron aportes en función de otorgar mayor calidad al presente estudio.

De igual modo se realizó la investigación documental para aportar elementos al escrito, realizando para ello la revisión de fuentes bibliográficas confiables que dieran insumos para desarrollar los antecedentes de investigación y las pautas para el análisis y profundización del tema de masculinidades en el territorio nacional.

\section{Antecedentes}

El trabajo para la prevención y mitigación de la Violencia Basada en Genero, ha sido un eje que se ha venido desarrollando e impulsando tanto por diversas instancias, quienes han incluido dentro de las estrategias de trabajo para aportar a la equidad e igualdad entre mujeres y hombres, el trabajo dirigido hacia los actores masculinos, quienes ungen como representantes del sistema patriarcal en los hogares, comunidades y sociedad en general.

En el año 2015 la agencia internacional de Irlanda en Nicaragua, TROCAIRE, realizo a través del programa de prevención de la Violencia Basada en Género, la sistematización de una variedad de estrategias innovadoras para involucrar a niños y hombres. En este documento se habla de elementos vinculados a una guía que incluía los siguientes elementos:

Los cambios en los hombres contribuyen al empoderamiento de las mujeres y su acceso a derechos y oportunidades", también es importante que los hombres descubran que sus propias vidas y relaciones (pareja y familia) mejoran cuando renuncian al modelo de masculinidad patriarcal. Cambios en las creencias, actitudes, valores y comportamientos de los hombres benefician a todos, aunque de distinta manera (TROCAIRE, 2015) 


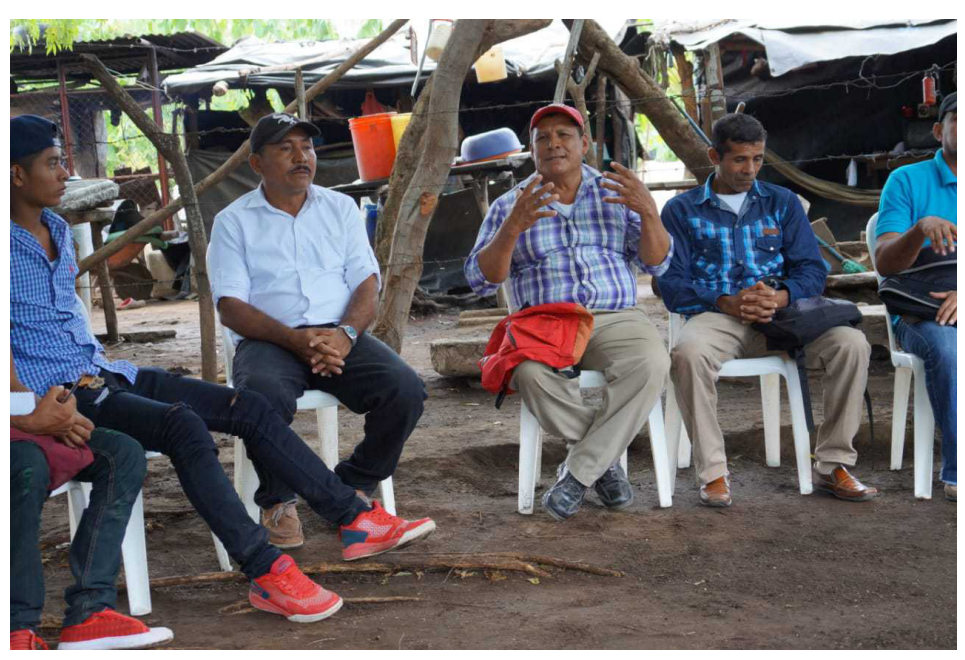

realizar la interrogante alusiva hacia el ¿Por qué del comportamiento violento de los hombres?, siendo el mismo comportamiento una práctica recurrente en el marco de las masculinidades hegemónicas donde se subordinan y violentan a las mujeres.

En este sentido las instancias que deciden trabajar la línea de la prevención de la Violencia Basada en Género, trataron de estructurar una especie de ruta lógica inducida del modo siguiente. “... Reconocerse, a hablar de ellos (hombres) y con ellos desde adentro y crear conciencia de que la violencia genera más violencia... iniciar un proceso permanente,

Existe mucha documentación que refleja los tipos de abordajes metodológicos en el trabajo con hombres y los que destacan la importancia de promover las buenas relaciones entre mujeres y hombres, trabajando desde los enfoque de educación popular en los contextos rurales, esto con la amplitud de opciones para concientizar, informar y prevenir futuras situaciones de violencia en las mismas comunidades.

Se ha enfatizado en la creación de grupos organizados de hombres como un medio para hablar y reflexionar sobre la importancia de cambiar aspectos de sus vidas que generan riesgos individuales y colectivos productos de asumir identidades temerarias y transgresoras asignadas por la misma sociedad, de esta forma estos hombres en niveles de cuestionamiento propositivo, pueden convertirse en referentes para otros hombres instándole a asumir roles beligerantes en la defensa de los derechos de las mujeres.

En el año 2003, La Asociación para la Investigación del Desarrollo Sostenible de Las Segovias ADESO "Las Segovias" realizo la sistematización titulada, masculinidad en positivo, en cuyas conclusiones logro reflejar los siguientes elementos. “...El trabajo de masculinidad es incipiente en el país, hay carencias de estrategias alusivas a este tema, los hombres por trayectorias históricas han marcados hitos como victimarios" (ADESO, 2003)

En relación a este último ámbito de los hombres vistos como potenciales victimarios, bien valdría la pena liberador, que incluya a hombres, mujeres, niños y niñas, jóvenes, en las distintas etapas" (UICN, San José, costa Rica, 1997)

No se puede perder de vista que el ejercicio de la masculinidad hegemónica emerge dentro del sistema patriarcal, mismo construido dentro de las esferas de relaciones sociales. Por tanto, partiendo desde el hecho de que la sociedad es cambiante, los sistemas hegemónicos pueden moldearse siempre y cuando se acompañe a los principales beneficiados de estos sistemas, para que puedan accionar hacia la elaboración de opciones novedosas para actuar como hombres regidos por el respeto, la afectividad sana y el buen trato.

A raíz de la investigación realizada en el 2003 por ADESO se destaca como uno de sus principales hallazgos. "La violencia es una acción conjunta que involucra a la familia en particular y sociedad en general. Para lograr su mitigación, se requiere de transformaciones globales inmediatas, a mediano y a largo plazo pudiendo iniciarse desde el espacio local" (ADESO, 2003)

Sin duda alguna se divisa los cambios en los hombres como la suma de distintos niveles donde el grado de mitigación se basa muchas veces en esfuerzos conjuntos y acciones que cada actor masculino ejerza en sus entornos de desarrollo, solo así pudiéramos hablar de un proceso de desconstrucción de las identidades masculinas, quizás no extremo y radical, pero si gradual e impregnado desde la misma cotidianeidad. 


\section{Resultados}

Previo a desarrollar los resultados del presente artículo se hará énfasis en relación a la categoría de "las nuevas masculinidades" misma que representa una variable amplia puesto que no siempre lo nuevo puede ser necesariamente bueno, bien pudiésemos estar ante una innovadora identidad desarrollada para infringir nuevas formas de sumisión y transgresión en el ejercicio de poder y roles desde hombres hacia mujeres, por ello se esclarece que para efectos de este escrito se entenderá la variable:

"La masculinidades en positivo", será entendida como aquella que define a la inmediatez el enfoque de productividad, eficiencia, respeto y afectividad sana en las relaciones entre mujeres y hombres, siendo la crítica hacia patrones de comportamiento hegemónicos, rescatando así las nuevas propuestas para vivenciar las masculinidades mediante opciones innovadoras y positivas realizadas desde los mismos hombres, siendo incluidas como un modelaje para la conducta de otros hombres en la sociedad actual.

\section{Masculinidades en positivo, una oportuni- dad de combatir el sistema patriarcal.}

En el presente artículo se pretende realizar un análisis sobre las expresiones de las masculinidades y la manera en las que se puede trabajar la prevención de la Violencia Basada en Género; al mismo tiempo trata de identificar algunas acciones que han sido implementadas en el país con el uso de herramientas metodológicas participativas.

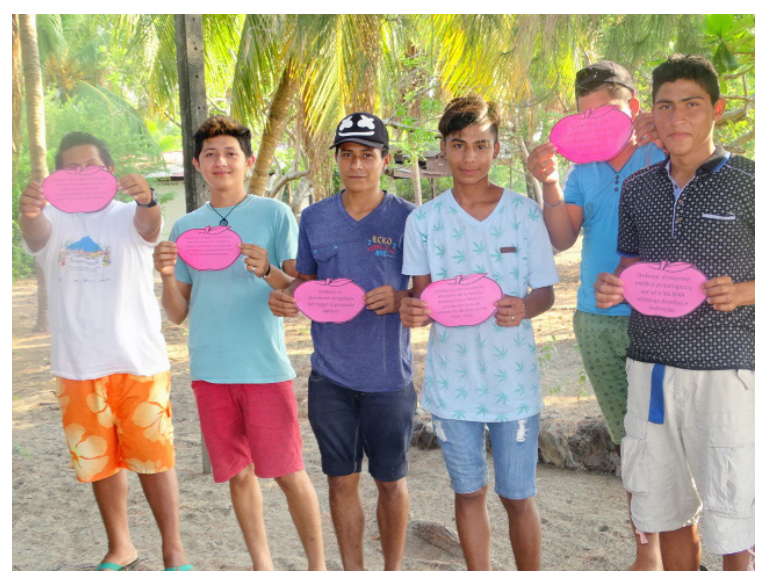

Tal como lo menciona, Welsh, P \& Piñón F "cuando hablamos de la masculinidad nos referimos a un conjunto de actitudes, valores y conductas consideradas aptas para los varones. Es un modelo histórico sociocultural de ser hombre que se construye sobre la base del sexo biológico". (Wesh, 2003).

Las nuevas masculinidades en positivo tienen como reto identificar momentos y elementos claves en la construcción social de las identidades masculinas para evidenciar aquellas prácticas abusivas de poder e identificar Violencia basada en el género y Violencia generacional en las familias y la sociedad nicaragüense; de esta forma se podrá aportar en la búsqueda de nuevas propuestas metodológicas para continuar con el trabajo de prevención de violencia por parte de los hombres. Claro está que la prevención es un escalón amplio que solo tiene cabida al momento de dimensionar la problemática como un problema real y significativo en la sociedad, por ello se inicia trabajando en función de informar acerca de las definiciones, expresiones, justificación y efecto que tiene la Violencia Basada en Genero (De ahora en adelante VBG) y Violencia Generacional (De ahora en adelante VG).

Partiendo de la definición de la VBG para algunos hombres que fueron entrevistados en el presente estudio destaca la siguiente definición por parte de uno de los líderes comunitarios. "La VBG es aquella que se hace a las mujeres, por ejemplo cuando la golpeas porque te desobedeció en alguna orden o porque andas borracho" (Líder comunitario, comunidad El Congo, 2018)

La cita anterior muestra una interesante definición de la VBG debido a que en la misma se incluyen elementos relacionados al reconocimiento de un tipo de violencia a como en efecto lo es la Violencia Física, sin embargo la misma VBG es mucho más amplia puesto que daña lesivamente las dimensiones físicas, emocionales, patrimoniales en las mujeres. Al mismo tiempo se notan justificantes de la VBG hacia las mujeres, siendo en el caso concreto en la cita del informante, la presencia de alcohol y demás sustancias controladas.

El análisis de la cita del informante tiende a ser un patrón en las respuestas emitidas por parte de los entrevistados, quienes de forma inconsciente tienden a normalizar la violencia o bien no hacen el análisis con las dimensiones reales e impactos a diversas escalas que 
la misma tiene. Sin embargo todo esto tiene una explicación lógica y es precisamente la falta de desapego por una posición ventajosa en la cual los hombres han sido colocados desde una lógica androcéntrica a lo largo de la historia.

Algunos efectos de este notorio androcentrismo se divisan a partir de aspectos claves en la sociedad como por ejemplo en la división sexual del trabajo, misma que evidencia la forma en la cual desde siglos pasados el trabajo de las mujeres han tenido que hacer un plus esfuerzo para ser reconocido, mas allá de su reclusión en el ámbito privado, siendo socialmente aceptado que los hombres participaran más activamente que las

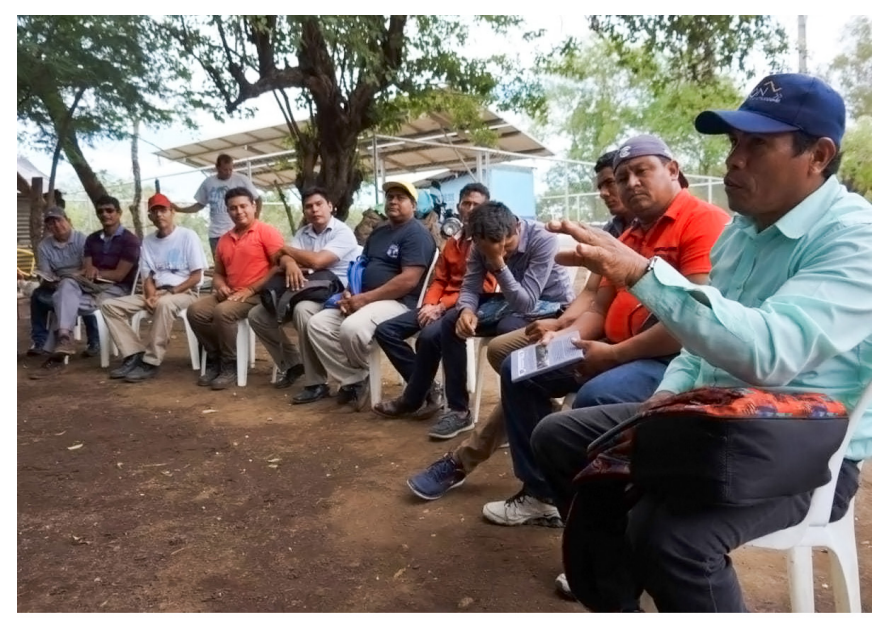
mujeres en un ambiente público, incluyendo los asuntos del Estado, de la economía y la producción de artes y ciencias.

La transmisión del conocimiento se hacía desde visiones totalmente masculinas, de hecho algunos espacios tales como los negocios o la política quedaban siempre reservados al grupo de varones y aunque algunas mujeres destacaban por su capacidad de generar una nueva visión o forma de generar nuevos conocimientos, lamentablemente siempre fueron tratadas y consideradas como "excepciones" (Garrocho, F 2012). Si bien es cierto hablar del tema de género ha sido una visión y trabajo de lucha por lograr la igualdad entre mujeres y hombres desde la primera ola del feminismo en el siglo XIX; el hecho de hablar por las masculinidades es relativamente más reciente.

El aporte desde la prevención es de gran relevancia para crear estrategias de trabajo en diferentes sectores tal como lo menciona Hernández en su artículo de "Estudios sobre Masculinidades, Aportes desde América Latina":

"Que los trabajos realizados a finales de la década de los noventa en Latinoamérica, coinciden al analizar la producción de la masculinidad e identidad masculina en relación al llamado "modelo de masculinidad hegemónica" o "modelo normativo de masculinidad" es por eso que se proponían ejes temáticos como construcción de la identidad masculina, la paternidad, los ámbitos de las relaciones entre la salud reproductiva y la sexualidad masculina" (Hernández, 2017)

Los ejes temáticos de paternidad responsable, los derechos sexuales y derechos reproductivos así como el propio tema de la identidad sexual, representan en muchos hombres, elementos particulares de la identidad masculina, por lo cual abordarlos en el marco de los procesos de reflexión y formación suele ser una línea base en este sentido.

Unicef (2004) en su libro sobre "Masculinidades y desarrollo social-Las relaciones de género desde las perspectivas de los hombres" comenta que:

Las publicaciones sobre los hombres como seres marcados por el género sólo comenzaron a realizarse en América Latina desde finales de la década de los ochenta. Hasta ese momento, la identificación de los varones con lo humano, y con una serie de privilegios hacía invisible su problemática de género (Unicef, 2004)

Teniendo en cuenta lo anterior podemos recalcar lo reciente que es el trabajo con hombres para la prevención de Violencia Basada en el Género y Violencia Generacional; sin embargo eso no ha sido obstáculo para la construcción de guías reflexivas y manuales que abordan diferentes ejes temáticos con metodologías participativas y vivenciales, las siguientes citas describen las propuestas en Nicaragua.

Welsh, P \& Muñoz, X en su guía elaborada en el año 2004, expresa que para reflexionar sobre hombres de verdad o la verdad sobre los hombres realizada en Nicaragua; es necesario construir propuestas metodológicas 
que potencien y faciliten procesos. Además menciona que la metodología de educación popular, en particular, aplicada a la propia subjetividad, es de suma importancia para facilitar la desarticulación de actitudes, valores y comportamientos machistas, y la construcción de alternativas y alianzas basadas en la equidad de género.

Por otra parte el Fondo de Población de las Naciones Unidas-UNFPA, en el año 2003, publica el manual para facilitadoras y facilitadoras; desde el proyecto de promoción de género y la participación masculina para adolescentes y jóvenes en cual propone sesiones para trabajar derechos sexuales y reproductivos para la prevención en diferentes niveles.

Si bien es cierto existen más instancias interesadas en trabajar la prevención de la violencia con hombres, las cuales diseñan proyectos con ejes temáticos centrados en las nuevas formas de masculinidades con hombres en las zonas urbanas y rulares del país; aún quedan muchos desafíos entorno a las estrategias de trabajo a utilizar en los diferentes contextos nacionales.

Podemos decir que hablar de masculinidades, además de ser un desafío actual, nace a raíz de la necesidad de crear nuevas pautas de vivir las masculinidades desde la condición de hombres, con enfoque de derecho y género; donde se fomente el respeto y la equidad para la prevención de Violencia Basada en el Género.

El trabajo de prevención debe estar asociado al uso de metodologías participativas, populares, vivenciales e inclusivas, con dicho fin de revindicar los derechos de las mujeres, recordando para ello que la mujer debido a su condición humana ya es sujeta de derecho y como tal debe ser trata en cada esfera de escenario social.

\section{Mitos y estereotipos de la masculinidad hegemónica en los contextos rurales.}

En el trabajo con hombres en Nicaragua, fueron discutidos ámbitos de género, masculinidad y violencia, en aquel entonces el análisis principal desarrollado por organismos internacionales y nacionales versaba sobre la credibilidad de trabajar en función de estos procesos.

La interrogante versaba en la posibilidad de pensar que en Nicaragua se lograse dejar de ver a los hombres como adversarios y contrario al desarrollo de la mujer, logrando integrarles hacia un propósito que demandaba sus niveles excesivos de altruismo puesto que su integración voluntaria representaba un gran reto por realizar compromisos políticos y dejar atrás los privilegios y poder otorgados por un sistema patriarcal.

Es por ello que Patrick Wesh en su libro "Los hombres no son de marte" expresa que el acto por dejar de figurar como representación autoritaria es. "Un gesto altruista que parecía diametralmente opuesto a todo lo que se entendía respecto al carácter dominante, controlador y autoritario de la masculinidad tradicional" (Wesh, 2000)

Al hablar de las figuras de poder y el control hegemónico desde las masculinidades tradicionales no se puede dejar aislada la fuerza que incluye el imaginario social, mismo que gesta una amalgama de mitos que refuerzan los ejercicios desiguales de poder entre mujeres y hombres, justificando al mismo tiempo los efectos de estas inequidades y desigualdades socialmente pronunciadas, asignadas y asumidas.

A raíz de la implementación de un instrumento cuantitativo - test, fue posible extraer algunos pensamientos recurrentes por parte de los hombres en relación a situaciones que ameritan enfoque de género y preventivo en contra de situaciones de violencia, algunas de las afirmaciones más recurrentes en los hombres entrevistados se enfocaron en los ámbitos de: Responsabilidad en la salud sexual - prevención de ITS, derechos sexuales y reproductivos y planificación familiar.

Los ejes descritos son inmersos en un total de 10 afirmaciones aplicadas hacia 63 hombres, en ellas se proyectan imaginarios sociales, estigmas y estereotipos que reafirman un sistema machista hegemónico desventajoso para las mujeres y su salud sexual y reproductiva, en relación a los resultados del ámbito asociado a la Salud Sexual, destaca lo siguiente

El 71\% (45) hombres responsabilizan a las mujeres
como las que tienen que prevenir las enfermedades de
transmisión sexual; el 84\% (53) hombres considera
que la planificación familiar es responsabilidad de
las mujeres, la mayoría $71 \%$ (45) hombres afirman
que el uso de preservativos es en relaciones con mu-
jeres que no son la esposa o compañera" (Datos, ex-
traídos, aplicación perfil de entrada, hombres, 2018) 
Los datos resultantes de la aplicación del instrumento tienen relación con lo Sherry Ortner retoma en la obra "Antropología y Feminismo", misma en donde la autora hace el planteamiento de que: Es la mujer con respecto al hombre, lo que es la naturaleza con respecto a la cultura, especificando a su vez que. "El cuerpo de la mujer parece condenarla a la mera reproducción de la vida... El determinismo biológico de la mujer les hace ser naturalmente subordinada" (Ortner, 1990).

Según lo planteado por la autora y a raíz de las afirmaciones realizadas por los informantes, es obvia una subordinación de la mujer en los escenarios maritales o relaciones de pareja, por tanto la masculinidad hegemónica responsabiliza a la mujer por las labores de carácter exclusivo en la reproducción.

De esta forma el hombre está aprovechando su condición de "superioridad social y biológica" para decidir su modo de disfrutar en plenitud su sexualidad aun cuando el costo de ello sea exponer a las féminas a recurrentes afectaciones a la salud sexual y la salud reproductiva.

Los efectos del sistema patriarcal han sido tan lesivos y mutiladores que incluso han relegado el empoderamiento de la mujer y la restitución de sus derechos sobre su propio cuerpo, se les veta la opción por decidir por sí mismas y para sí mismas, producto de las relaciones de poder y sumisión a las cuales se ven sometidas.

Uno de los grandes éxitos del sistema patriarcal ha sido entre muchos otros, la cosificación de las mujeres por parte de los hombres en la sociedad, este éxito sin duda alguna es parte de los mitos y estigmas que aún persisten en la sociedad y se evidencia en las afirmaciones realizadas por parte de los informantes claves del presente estudio, quienes expresan.

"El 67\% (42) hombres consideran que las mujeres deben tener el permiso de la pareja para poder atender su salud sexual y reproductiva. Mientras el 78\% (49) hombres consideran que el hombre tiene derecho de decidir sobre cuantas hijas (os) debe tener la mujer" (Dato - aplicación perfil de entrada, hombres, 2018)

Tal como lo expresar Ortner, un resultado del sistema es el eficiente Feedback:

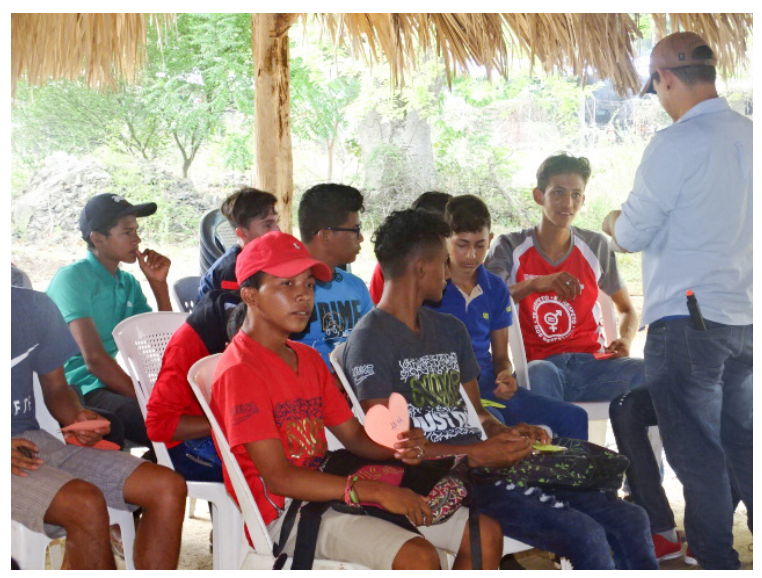

Los distintos aspectos (físicos, sociales, psicológicos) de la situación de la mujer, colaboran a que sean más vistas como próxima a la naturaleza donde los roles de procreación, son claramente impuestos y no negociables, el hombre en su posición clásica es quien decide la condición de derecho de la mujer y su "bienestar (Ortner, 1990).

Decidir sobre el cuerpo y la salud de las mujeres, son señalares alarmantes de los niveles de sumisión a los cuales las mujeres están sujetas y al mismo tiempo evidencian la falta de negociación en la satisfacción de necesidades básicas como la sexualidad y reproducción.

A tal grado de que el hombre impone incluso la frecuencia de los encuentros sexuales, amparándose del muy reforzado mito que expresa, que la mujer siempre tienen deseos sexuales sin importar la condición en la que esta se encuentre, la afirmación en este sentido es fuerte debido a que. "El 70\% (44) hombres no reconocen el derecho de las mujeres a decidir cuándo tener o no tener relaciones sexuales" (Dato - aplicación perfil de entrada, hombres, 2018)

Es una necesidad latente al desconstruir masculinidades clásicas que incluye la desmitificación de la condición temeraria del hombre, dejar de creer que el hombre por su condición social y biológica es un ser que además de reprimir y maltratar de diversas formas es casi inhume al peligro o los factores de riesgo a su condición de bienestar integral, esta percepción social del hombre se hace expresa en afirmaciones de este tipo. "El $81 \%$ (51) hombres consideran que los 
hombres no necesitan cuidar de su salud sexual y reproductiva" (Datos, extraídos, aplicación perfil de entrada, hombres, 2018)

Este y los demás resultados del instrumento dirigido permiten identificar de formar general el alto porcentaje de hombres que reafirmar y defienden los mitos y creencias patriarcales en las relaciones maritales y sociales entre mujeres y hombres. La lectura a los datos extraídos en la aplicación apunta una diversidad de análisis.

Entre los análisis resultantes destaca que la mayoría de los hombres no identifican su responsabilidad ni consideran necesitarlo; sino que responsabilizan a las mujeres del contagio de las ITS - VIH y aunque el hombre piense que las mujeres tienen derechos de decidir sobre ellas; en la percepción de la mayoría de los hombres no se reconoce el derecho de la mujer de decidir sobre su propio cuerpo.

Los hombres además justifican bajo distintas circunstancias la violencia sexual ejercida desde ellos hacia otras mujeres, en cuanto a la planificación familiar es vista por la mayoría de hombres como una carga que deben asumir las mujeres y atribuyéndose por otro lado el hombre el derecho de decidir la cantidad de hijos e hijas que debe tener la mujer, alegando que se debe tener los hijos e hijas que Dios manda.

Es claro que muchos de los conocimientos e ideas expresadas por los hombres, son basados en mitos y creencias machistas que parten de la ideología de la superioridad del hombre, sobre la mujer, atribuyéndose el derecho de decidir sobre la vida de las mujeres, descartando antagónicamente la necesidad de cuidar de su propia salud.

Esto hace oportuna la importancia por implementar intervenciones que promuevan la sensibilización y concientización en materia de genero desde la propuesta de las masculinidades en positivo, de este modo los hombres podrán reconocer su clara ventaja y complicidad sobre la opresión de las mujeres, para en un segundo momento poder brindar el reconocimiento a las féminas como sujetas de derechos y no como objetos de propiedad privada.

\section{El enfoque de las masculinidades en positivo, una puerta para la promoción del desarrollo integral en las familias.}

Al retomar el trabajo de las masculinidades en positivo no solo se está en función de promover cambios en el hombre desde su condición individualizada, sino también desde sus relaciones creadas con las mujeres, niñas, niños, adolescentes, jóvenes y otros hombres.

La intención es crear agentes de cambios positivos y protectores en las familias, dejando relegado los roles que incitan a ser factores de riesgo para sí mismos y otras personas, de este modo también pasarían a ser actores claves y referentes de positivos en sus comunidades.

Los hombres al ser abordados desde el enfoque de las nuevas masculinidades en positivo son instados a reconocer la importancia de su integración a procesos de cambios, como una opción en donde se auto reconozcan como hombres activos en sus roles y dinámicas habituales, quienes pueden dedicarse tiempos, promover la afectividad sana entre sus pares sin temor a un cuestionamiento incluso hacia su orientación sexual.

Una etapa de cambio que parte desde las dimensiones intrapersonales, hacia las interpersonales, la cual insta hacia la desmitificación de fraseos que señalan cambios por parte de los hombres en pro de otras personas como por ejemplo: "Lo hago para ayudarle", "yo le ayudo a mi mujer cuando está enferma", "Hay que cambiar por las mujeres"; los hombres deben comenzar a realizar los cambios por ellos mismos, solo así los cambios serán realmente personales, políticos y con sentido de practicidad en tiempos coherentes con lo que la sociedad realmente demanda, a como en efecto lo es el cuido y preservación de la vida de las mujeres.

Las acciones de intervención social y comunitaria deben ser inclinadas hacia cambios graduales y no radicales, de esta forma se estaría consciente de lo que realmente se quiere y se puede lograr con el trabajo hacia los hombres, sobre todo considerando que se está cuestionando un sistema patriarcal en donde ellos figuran como los principales benefactores, por tanto no se puede obviar que el hecho de renunciar a las ventajas del sistema no es una opción alentadora que pueda tomar a la ligera para muchos hombres. 
Patrick Wesh también expresa que "Para minimizar los riesgos y maximizar los beneficios para hombres y mujeres, el trabajo en género y masculinidad debe continuar desarrollándose en un marco pro feminista que defina la igualdad de género como su última meta" (Wesh, 2000), claro está que el fin mismo de un proceso no es perpetuar una justificación activa en donde los hombres retarden cambios necesarios para aportar hacia la equidad de género.

En otras palabras los hombres no puede alegar de forma vitalicia que la reincidencia en sus acciones con carácter machista, se deben a que están inmersos en un proceso de conciencia o sensibilización aun no finalizada y por ello sus actos deben ser tolerados incluso conforme se aglutinen los años.

No se puede obviar que el involucramiento de los hombres en los procesos de cambios parte de un discurso y se ve aún más reforzado con su sentido práctico, es esa coherencia la que determinara si su involucramiento es realmente una alternativa de cambio o solamente se remonta hacia una falacia o utopía constante y reincidente.

$\mathrm{Al}$ respecto Patrick Wesh definen en su obra, "los hombres no son de marte", que "Son los hombres quienes deben tener la última responsabilidad en promover y consolidar los procesos de cambios en sí mismos y en otros hombres. Al hacerlo, deben reconocer que el aporte crítico de las mujeres... no solo deseable sino indispensable" (Patrick Welsh, 2000)

Los llamados procesos de cambios positivos, no es una labor aislada de solo un segmento de la población o al menos no debería ser vista de ese modo, las mujeres deben reconocer que el empoderamiento es una primicia que les corresponde, en donde el respaldo de los hombres es clave para alcanzar niveles reales de cambios, así mismo el hombre debe reconocer que la mujer en su propósito de ser resiliente, adquirir conciencia y volverse crítico del sistema, es por mucho la mejor aliada que pueden tener las figuras masculinas para salir adelante promoviendo la equidad e igualdad entre ambos sexos.

El aporte de las organizaciones de mujeres y de las instancias del estado son de igual forma elementos determinantes que permiten el desarrollo integral en la sociedad, para ello hay muchos avances en materia legislativa, puesto que Nicaragua es sin duda uno de los países a nivel latinoamericano con un vasto marco legal en pro de la prevención y sanción de la VBG y VG

El desafío siguiente radica en implementar a cabalidad y conciencia lo que la ley dispone, por ello no debemos ser permisivos ante situaciones de abusos, ni promover la complicidad masculina en ello, puesto que es ahí donde realmente está la muestra de cambios, en la no aceptación, la argumentación del rechazo hacia practicas añejas y transgresoras y la nuevas propuestas para relacionarse de manera sana y respetuosa por parte de los hombres hacia las mujeres.

Wesh expresa que "Para maximizar la contribución de los hombres a la equidad de género es importante que las iniciativas sobre masculinidad tengan lugar dentro de un amplio marco de género en el desarrollo, que se centre en el desarrollo integral de todas las mujeres y hombres y en mejorar la calidad de sus vidas y de sus relaciones. (Patrick Welsh, 2000)

Es decir ampliar el enfoque, reconocer que las posturas radicales no son necesariamente la mejor condición para adquirir un estado real de derecho, claro está que el accionar no solo de Nicaragua, sino también de América Latina debe llevarse a cabo siguiendo un lineamiento teórico especifico, puesto que ese elemento determinaría las acciones a desarrollar por parte todos los sectores sociales involucrados en esta problemática que afecta a traves de distintas intersecciones a toda la población en general.

\section{Conclusiones}

El enfoque de trabajo con hombres debe ser definido bajo la línea de trabajo de masculinidades en positivo, de esta forma podrán ser definidas una amalgama de acciones y propuestas que puedan transformar a los hombres lejos de sus papeles como factores de riesgo y más como agentes positivos de cambio.

Podemos decir que hablar de masculinidades es un desafío actual y que nace a raíz de la necesidad de crear nuevas pautas de vivir las masculinidades desde la condición de hombres, mismos con enfoque de derecho y género; donde se fomente el respeto y la equidad para la prevención de Violencia Basada en el Género El modus vivendus de muchos hombres y su imaginario social está viciado por mitos y creencias machistas 
que parten de la ideología de la superioridad del hombre sobre la mujer, atribuyéndose el derecho de decidir sobre la vida de las féminas, descartando la necesidad de cuidar de su propia salud.

Al trabajar bajo el enfoque de las nuevas masculinidades en positivo se hace oportuna la necesidad de implementar intervenciones que promuevan la sensibilización y concientización en materia de genero para brindar el reconocimiento a las féminas como sujetas de derechos y no como objetos de propiedad privada. Retomar el trabajo de las masculinidades en positivo no solo se está en función de promover cambios en el hombre desde su condición individualizada, sino también desde otras relaciones con las mujeres, niñas, niños, adolescentes, jóvenes y otros hombres.
El involucramiento de los hombres en los procesos de cambios parte de un discurso y se ve aún más reforzado con su sentido práctico, es esa coherencia la que determinara si su involucramiento es realmente una alternativa de cambio o solamente se remonta hacia una falacia o utopía constante y reincidente.

En Nicaragua y América Latina el trabajo de género y masculinidades, debe llevarse a cabo siguiendo un lineamiento teórico especifico que determinara las acciones a desarrollar por parte de todos los sectores sociales involucrados y quienes deben reconocer la problemática que implica aun la vigencia del sistema patriarcal para el desarrollo de una sociedad que desea proponer desde la equidad para la igualdad en derechos y oportunidades. 


\section{Referencia Bibliográfica}

Castillo, J (2003) "Masculinidades en Positivo", Estelí Nicaragua Recuperado de: Asociación para la Investigación del Desarrollo Sostenible de Las Segovias ADESO

Faur, E (2004) "Las relaciones de género desde la perspectiva de los hombres" Recuperado de: https://www.unicef.org/masculinidades.pdf

Fondo de Población de las Naciones Unidas-UNFPA, (2003), "Alternativas, queremos y podemos tener una vida mejor cuidando nuestras relaciones" Recuperado de:. https://nicaragua.unfpa.org/sites/ default/files/pub-pdf/Documento-Estrategia

Garrocho, F. (2012). "Educación y modelos de género en Europa durante los siglos xix y principios del xx: "el modelo de finlandia"1 y el nacimiento del feminismo europeo" [online] Institucional.us.es. Available at: http:// institucional.us.es/revistas/cuestiones/21/ art_16.pdf [Accessed 30 Aug. 2018].

Hernández, O (2007), "Estudios sobre Masculinidades. Aportes desde América Latina". Revista de Antropología Experimental. Recuperado de: http://americalatinagenera.org/newsite/images/cdr-documents/publicaciones/estudios_sobre_masculinidades_al.pdf.
PNUD (2005), Informe sobre desarrollo humano, Madrid, Ediciones Mundi Prensa.

Ortner S. X, (1973) "Antropología de América". Inglaterra. Recuperado de: publicaciones británicas, purificación, creencias y prácticas

TROCAIRE (2015) "Involucrando a los hombres para la eliminación de la violencia de género en Nicaragua". Managua, Nicaragua Recuperado de: www.trocaire.org

Welsh, P \& Muñoz, X, (2004) "Hombres de verdad o la verdad sobre los hombres". Managua-Nicaragua. Recuperado de: https://revistas.unimilitar.edu.co/index.php/rlbi/article/download/3388/2913

Welsh, P \& Piñón F (2003) "Apuntes para un Marco Teórico: Género y Masculinidades" Recuperado de: http://www.ilam.org/viejo/ILAMDOC/ museos.es/4-2008.pdf

Welsh, P (2001) "Los hombres no son de marte, desprendiendo el machismo en Nicaragua" Recuperado de: EC.DAPHNE PROGRAMME, 2001.

\section{Sergio José Hernández Briceño}

Licenciado en Antropología social, con post grado en investigación multidisciplinaria, especialidad en áreas de trabajo comunitario con enfoque de educación popular con sector adolescencia, juventud y adultez, empleando enfoque de intervención psicosocial, egresado de la carrera de derecho en la universidad Centroamericana (UCA), estudiante activo de psicológica con mención en clínica en la Universidad Autónoma de Chinandega (Uach), participante activo de las Jornadas Universitarias de Desarrollo Científico en la UNAN-Managua; ensayista destacado en concursos de tipo histórico y político en competencias interuniversitarias; distinguido orador en congresos y conferencias universitarias. 